\title{
Dynamics of Electrochemical Conversion of Nanoscale Metal-Metal Oxide Multilayer Architecture
}

Fernando C. Castro ${ }^{1}$, Qianqian $\mathrm{Li}^{2}$, Guennadi Evmenenko ${ }^{1}$, D. Bruce Buchholz ${ }^{1}$, Jinsong Wu ${ }^{1,2}$, Michael Bedzyk $^{1}$, Vinayak P. Dravid ${ }^{1,2}$

${ }^{1}$ Department of Materials Science and Engineering, Northwestern University, Evanston, IL, USA

2.NUANCE Center, Northwestern University, Evanston, IL, USA

Lithium-ion battery technology has progressed to the point where it is commonplace in today's consumer electronics. However, future applications also demand electrode materials with increased performance over typical intercalation-based electrodes. Electrode materials that undergo conversion reactions can offer this increased performance by storing larger amounts of energy compared to intercalation materials, but suffer from energy inefficiencies during electrochemical cycling. [1] Inefficiencies due to first-cycle irreversibility and charge overpotentials especially hinder in conversion reaction anodes made from $3 d$ transition metal oxides, as these anodes undergo significant structural changes during cycling. [2] Further structural characterization, especially under the dynamic conditions of applied bias, is necessary to understand the link between cycling behavior and energy inefficiencies found in the active material.

In this work, we have investigated microstructure evolution in conversion anodes with the goal of understanding the lithiation kinetics, ionic diffusion, and nucleation and growth phenomena. Anodes with architecture of alternating $\mathrm{Ni}$ and $\mathrm{NiO}$ layers are used, as seen in Figure 1. During electrochemical lithiation, $\mathrm{NiO}$ undergoes conversion and provides a charge capacity $>700 \mathrm{mAh} / \mathrm{g}$, doubling the charge capacity of traditional carbon-based intercalation anodes. Furthermore, these multilayer structures have been shown to accommodate volume expansion and improve cycling lifetimes in related Li-alloy materials. [3] However, the $\mathrm{Ni} / \mathrm{NiO}$ multilayer architecture also enables improved control over initial anode nanostructure by tuning structural parameters, e.g. layer thickness and organization.

We have analyzed the multilayer structures and the interfaces with Scanning Transmission and Transmission Electron Microscopy (S/TEM) techniques, coupled to complementary X-Ray Reflectivity (XRR) measurements. The combination of both approaches reveal different extents of the conversion reaction based on layer thickness and organization. Lithiation of multilayers with $5 \mathrm{~nm}$ layers of Ni show no $\mathrm{NiO}$ conversion past the top $\mathrm{NiO}$ layer (Fig. 1A), while multilayers with thinner Ni layers undergo more complete conversion. Examining bilayer $\mathrm{Ni} / \mathrm{NiO}$ films at the early stages of lithiation (Fig. 2) uncover the formation of interfacial Ni-containing reaction layers. S/TEM Electron Energy Loss Spectroscopy (EELS) was used to uncover the changing distributions of conversion reaction products throughout the electrochemical process. These results shed considerable light on the lithiation mechanism in the multilayer system and highlight changes in layer nanostructure that may impede Li transport and $\mathrm{NiO}$ conversion, resulting in overpotentials found in the system. The presentation will cover both static analysis of microstructure evolution and ongoing dynamic in-situ S/TEM lithiation experiments with such model multilayer systems. [4] 
References:

[1] P. Poizot et al., Nature 407 (2000), p. 496.

[2] L. Luo et al., Scientific Reports 4 (2014), p. 3863

[3] T.T. Fister et al., Advanced Energy Materials 4 (2014), p. 1301494

[4] This work was supported as part of the Center for Electrochemical Energy Science (CEES), an Energy Frontier Research Center funded by the U.S. Department of Energy, Office of Science, Basic Energy Sciences. This work made use of the EPIC facility of the NUANCE Center at Northwestern University, which has received support from the Soft and Hybrid Nanotechnology Experimental (SHyNE) Resource (NSF NNCI-1542205); the MRSEC program (NSF DMR-1121262) at the Materials Research Center; the International Institute for Nanotechnology (IIN); the Keck Foundation; and the State of Illinois, through the IIN.
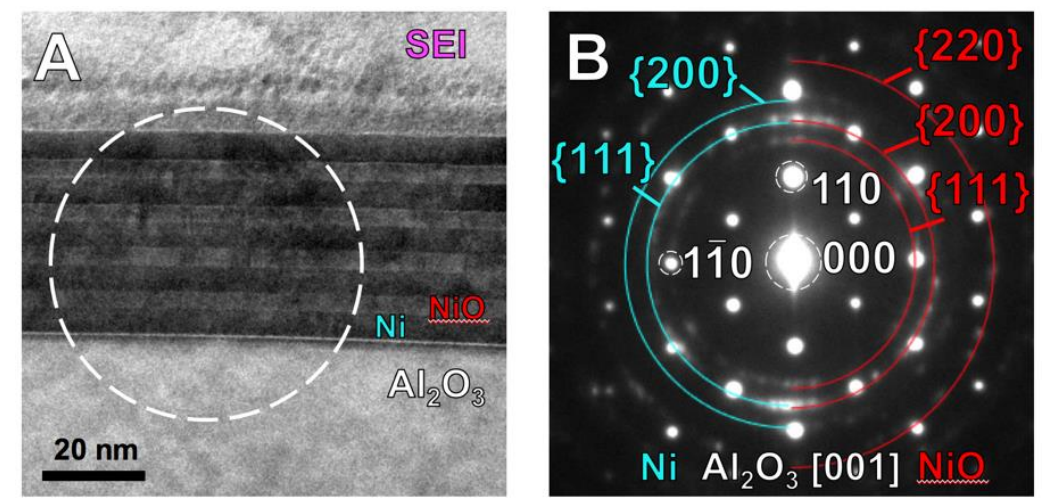

Figure 1. (A) HRTEM image of lithiated multilayer film with 5 bilayers of $5 \mathrm{~nm} \mathrm{Ni}$ and $5 \mathrm{~nm} \mathrm{NiO}$ on $\mathrm{Al}_{2} \mathrm{O}_{3}$ substrate. Only the top $\mathrm{NiO}$ layer has undergone lithiation as substantial Li transport did not occur through the $5 \mathrm{~nm}$ thick Ni layer. (B) SAED from area indicated by dashed circle in (A). NiO diffraction rings are clearly seen, indicating the absence of any $\mathrm{NiO}$ conversion reaction to $\mathrm{Ni}$ in the lower layers of the structure.
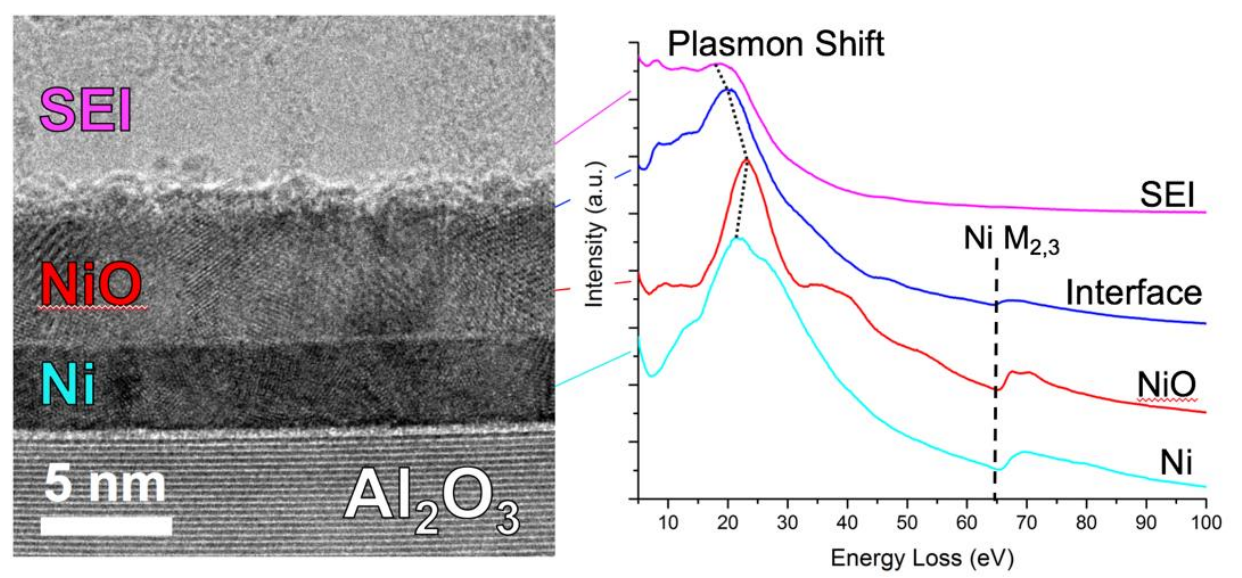

Figure 2. HRTEM image of Ni/NiO Bilayer Film on Al2O3 substrate, with EELS spectra collected in different regions of the bilayer architecture. A Ni-containing interface has formed on top of the $\mathrm{NiO}$ layer, revealing part of the reaction mechanism during the early stages of conversion. 